\title{
Determining the Sensitivity and Specificity of Electroneuromyographic Tests in Patients with the Prediagnosis of Ulnar Neuropathy at the Elbow \\ PD Analan ${ }^{1}$, B Leblebici $^{2}$
}

\begin{abstract}
Objective: The present study aimed to assess the sensitivity and specificity of nerve conduction studies (NCS) in patients pre-diagnosed with ulnar neuropathy at the elbow (UNE).

Method: This study assessed 166 extremities of 124 patients (68females, 56males) using electroneuromyographic (ENMG) testing. Nerve conduction velocity (NCV), sensory nerve action potential (SNAP) amplitudes, and compound motor action potential (CMAP) amplitudes were obtained by stimulating the ADM and FDI muscles. Patients were divided into two groups based on diagnosis made after ENMG testing; the UNE group (11 patients) and the non-UNE group (114extremities of 84 patients).The remaining 41 extremities (15 females and14 males) with non-UNE ENMG pathologies were excluded from the data set. Sensitivity and specificity values of the motor nerve conduction studies at the abductor digiti minimi(ADM) and first dorsal interosseous (FDI) muscles were determined. NCV of the forearm segment of the ulnar nerve was subtracted mathematically from the NCV of the elbow segment to determine the effectiveness of the forearmelbow inter-segmental velocity difference (FIVD)for the diagnosis of UNE.
\end{abstract}

Result: Agreement between the pre-diagnosis and the electrophysiological diagnosis of UNE was $6.7 \%$. Ulnar CMAP amplitudes of the ADM at the elbow segment and SNAP amplitudes at the elbow segment were similar (p>0.05).Reduced CMAP amplitude had $100 \%$ sensitivity and specificity. Sensitivity and specificity rates for NCV from the FDI were $99.1 \%$ and $81.8 \%$, respectively, and $98.2 \%$ and $90.9 \%$, respectively from the ADM. Sensorial, ADM and FDI FIVD sensitivity rates were $81.8 \%, 81.8 \%$, and $72.7 \%$, respectively, and specificity rates were $86.8 \%, 78.1 \%$, and $90.2 \%$, respectively.

Conclusion: Agreement between clinical pre-diagnosis and ENMG diagnosis of UNE was very low. However, the determination of reduced amplitude and NCV in the elbow segment of the ulnar nerve using ENMG had high sensitivity and specificity. In such patients, FIVD may be useful in diagnosing UNE.

Keywords: Elektroneuromyography, sensitivity and specificity, ulnar neuropathy at the elbow

From: ${ }^{1}$ Department of Physical Medicine and Rehabilitation, Faculty of Medicine, Baskent University ${ }^{2}$ Adana Research and Education Center, Adana, Turkey.

Correspondence: Dr PD Analan, Department of Physical Medicine and Rehabilitation, Faculty of Medicine, Baskent University, Kazım Karabekir Cad 4227 Sok. No 37 Yüreğir, Adana, Turkey. Fax: 00903223444445, e-mail: doruk.pinar@gmail.com 


\section{INTRODUCTION}

The ulnar nerve enters the ulnar groove formed between the medial epicondyle and the olecranon process at the elbow. The ulnar nerve travels slightly distal to the groove in the proximal forearm under the tendinous arch of the two heads of the flexor carpi ulnaris (FCU) muscle, known as the cubital tunnel. Entrapment of the ulnar nerve in this region is known as ulnar neuropathy at the elbow (UNE) (1).

In patients with UNE the clinical picture ranges from intermittent paresthesias in the fourth and fifth digits to complete sensory loss in the territory of the ulnar nerve with atrophy and weakness of the ulnar muscles. As the majority of the intrinsic hand muscles are ulnar innervated, weakness of these muscles leads to loss of dexterity and decreased grip and pinch strength $(1,2)$. UNE is the second most common entrapment neuropathy after carpal tunnel syndrome (CTS).

Electroneuromyographic (ENMG) tests are commonly used for the diagnosis of UNE. In addition, ENMG can be used to determine nerve dysfunction and its severity (1).In 1999 the American Association of Electrodiagnostic Medicine (AAEM) published ENMG application protocols for UNE $(3,4)$. These ENMG protocols include sensory and motor nerve conduction studies (NCS) and needle electromyography (EMG) applications. Motor NCS can be made by recording from the abductor digiti minimi (ADM) and/or first dorsal interosseous (FDI) muscles with ulnar nerve innervations (1). No international agreement has been made concerning the sensitivity and specificity of electrodiagnostic studies for the diagnosis of UNE. Sensitivity rates have been reported between $37 \%$ and $86 \%$ and specificity starting at $95 \%$ (2). However, motor NCS tests have shown different sensitivities and specificities in the literature $(2,4,5,9,12,13,14)$. Some studies have reported that ENMG of the FDI is more sensitive, others have suggested that ADM recordings alone are adequate and yet others advocate taking both recordings. Despite these various application possibilities, 
diagnosis may not be verified in some patients. The results of one electrodiagnostic study showed that $16 \%$ of patients with electrophysiological UNE were not diagnosed in evaluations using only two stimulation sites below and above the ulnar groove(5).

The present study aimed to assess the sensitivity and specificity ENMG studies in patients pre-diagnosed with UNE.

\section{METHOD}

This study was approved by the Local Ethics Committee and written informed consent was obtained from all participants. Power analysis during the bio-statistical preliminary assessment indicated a study population of 137 patient extremities. Consequently, this prospective study included 166 upper extremities of 124sequential patients (68 females and 56 males) referred to our ENMG Clinic.

Inclusion criteria were clinical pre-diagnosis of UNE in addition to the presence of paresthesia, weakness or pain in the ulnar nerve territory which worsened or became more prominent at elbow flexion, and positive Tinel's sign at the ulnar groove.

Exclusion criteria were neurological conditions such as stroke, focal or generalized neuropathies, brachial plexus lesions, lesions of the ulnar nerve proximal or distal to the elbow, a history of upper extremity surgery, trauma or corticosteroid injections for treatment of these symptoms, and inflammatory or rheumatologic disorders that potentially affected nerve conduction such as rheumatoid arthritis or gout. Pregnancy, systemic disorders affecting peripheral nerves such as diabetes mellitus or chronic renal failure, and any abnormalities found via NCS were also reasons for exclusion.

Patients were divided into two groups based on diagnosis made after ENMG testing. The UNE group included 11 patients (6females and 5 males)and the non-UNE group 
consisted of 114extremities of 84 patients (47 females and 37 males).The remaining 41 extremities of 29 patients (15 females and 14 males)who had non-UNE ENMG pathologies were excluded from the data set and statistical evaluation was performed based on the two groups.

\section{Nerve Conduction Studies}

All tests were performed using a four-channel Medelec Synergy ENMG system (Oxford Instruments Medical, Surrey, England).

If the hands of the patients were cool they were warmed by a hot pack before testing. Patients were seated on an exam chair and a pillow placed on the lap to relax the hands. Motor NCS was performed using superficial disc electrodes $(\mathrm{Ag} / \mathrm{AgCl})$ of $10 \mathrm{~mm}$ diameter and sensory NCS using ring-shaped recording electrodes. Stimuli were provided by bipolar electrodes with a distance of $25 \mathrm{~mm}$ between the ends and $6 \mathrm{~mm}$ diameter. The distance between active and reference electrodes in all ENMG evaluations was $4 \mathrm{~cm}$. A metal ground electrode was placed between the active and stimulating electrodes.

Motor orthodromic and sensory antidromic studies of the ulnar and median nerves were performed on the extremities of complaint of all patients included in the study. In addition, median nerve palm-wrist segment NCS were performed in order to assess isolated UNE. In patients with non-UNE ENMG pathologies, further ENMG testing was scheduled for diagnosis. Ulnar NCS was performed with the elbow at $70-90^{\circ}$ flexion in order to prevent subluxation of the nerve in the ulnar sulcus. For motor nerve-conduction studies, the recording electrode was placed at the belly points of the ADM and FDI muscles. The reference electrode for the $\mathrm{ADM}$ stimulus was placed immediately distal to the $5^{\text {th }}$ metacarpophalangeal (MCP)joint and at the middle of the thumb for the FDI. Stimuli were given from the wrist, $4-6 \mathrm{~cm}$ below and $6-8 \mathrm{~cm}$ above the midpoint of the ulnar sulcus at the 
elbow, the axilla and at Erb'sPoint. When giving stimulus from the wrist to the ADM, the distance between the recording electrode was adjusted to $8 \mathrm{~cm}$. The stimulation of the FDI from the wrist was performed over the FCU tendon at the proximal wrist line level.

the ulnar sensory NCS, the cathode ring electrode was placed at the $5^{\text {th }}$ MCPjoint and the anode electrode at the $5^{\text {th }}$ distal interphalangeal joint. The distance between the two points was arranged to give stimuli from $12 \mathrm{~cm}$ proximal to the cathode ring electrode at the proximal. The other stimulation points were located $4-6 \mathrm{~cm}$ below and $6-8 \mathrm{~cm}$ above the midpoint of the ulnar sulcus at the elbow.

In the median NCS, the sensory latency was recorded from the third MCP joint stimulated at a point $12 \mathrm{~cm}$ proximal to the recording electrode. Motor NCS was measured from the mid portion of the abductor pollicis brevis(APB) muscle and by the stimulation of the median nerve $8 \mathrm{~cm}$ proximal to the recording electrode. Proximal stimulations were performed in the medial epicondylar area.

For the median palm-wrist segment NCS, the active superficial disc recording electrode was placed at the proximal palm-wrist segment line and the reference electrode was placed at $4 \mathrm{~cm}$ proximal to the active electrode. The anode part of the stimulator was placed on the $2^{\text {nd }} \mathrm{MCP}$ joint and the cathode was placed in the palm to face the wrist.

Mixed, motor and sensory nerve conduction velocities (NCV), motor distal latencies (MDL), sensory distal latencies, compound muscle action potential (CMAP) amplitudes, sensory nerve action potential (SNAP) amplitudes, and F wave latencies were recorded.

For sensory distal latencies, the point at which the SNAP amplitude peaked was taken as the reference and the first separation point from the isoelectric line at the negative direction for the MDL. For the CMAP and SNAP amplitudes, the measurement between the first negative peak point of the action potential and the isoelectric line was recorded. 
The same type and size of electrodes were used for all patients. Filter settings were $3 \mathrm{HZ}$ to $10 \mathrm{kHZ}$ for motor NCS and $20 \mathrm{HZ}$ to $2 \mathrm{kHZ}$ for sensory NCS. In order to obtain the most optimal result in the NCS, all stimuli were given supramaximally. To minimize technical factors and optimize the results a minimum of 8 recordings were obtained for sensorial NCS. The average of these results was accepted as the mean result. For motor NCS, at least 3 recordings were obtained and the best result recorded.

\section{Electrodiagnostic criteria}

Diagnoses were made using AAEM recommendations (3) and parameters developed in the local ENMG laboratory.

Accordingly, for stimulus from the ADM muscle the following values were considered normal: ulnar nerve MDL $<3.45 \mathrm{~ms}$; CMAP amplitude $>4.32 \mathrm{mV}$; NCV $>53.6 \mathrm{~m} / \mathrm{s}$; F wave latency $<28.41 \mathrm{~ms}$; ulnar nerve sensory distal latency $<3.41 \mathrm{~ms}$; SNAP amplitude $>11 \mathrm{mV}$; NCV $>54.2 \mathrm{~m} / \mathrm{sn}$. With stimulus from the FDI muscle, stimuli limits were determined as follows: MDL<3.40 ms, CMAP amplitude $>4.30 \mathrm{mV}, \mathrm{NCV}>54 \mathrm{~m} / \mathrm{s}$.

For the median nerve, the following results were assumed as normal with stimulus from the APB muscle: MDL<4.00 ms, CMAP amplitude $>4.25 \mathrm{mV}, \mathrm{NCV}>50.3 \mathrm{~m} / \mathrm{s}$, sensory distal latency $<3.41 \mathrm{~ms}$, SNAP amplitude $>10 \mathrm{mV}, \mathrm{NCV}>50.6 \mathrm{~m} / \mathrm{s}$, mean F wave latency $<27.87 \mathrm{~ms}$. In the median nerve mixed NCS, NCV at the palm-wrist segment of $>35.9 \mathrm{~m} / \mathrm{s}$ and distal amplitude> $32.4 \mathrm{mV}$ were considered normal.

Electrodiagnostical criteria for UNE as measured by the motor NCS were as follows: $\mathrm{NCV}$ at the elbow segment of the ulnar nerve $<50 \mathrm{~m} / \mathrm{s}$, amplitude $<20 \%$ that of the amplitude obtained with stimulus from the wrist, mean F wave latency $>28.41 \mathrm{~ms}$, sensory $\mathrm{NCV}<48$ $\mathrm{m} / \mathrm{s}$, and SNAP amplitude $<2 \mathrm{mV}$. Amplitude of the elbow segment $20 \%$ lower than the 
forearm segment was considered conduction block and reduced NCV of the same segment focal demyelination.

Differences between the NCV of the forearm and elbow segments were evaluated as reported in Shakir et al. (6). The forearm-elbow inter-segmentary velocity difference (FIVD) was calculated for both sensory and motor NCS as the NCV of the forearm segment was mathematically subtracted from the NCV of the elbow segment. Needle electromyography (EMG) was applied to the FCU, ADM, and FDI muscles of patients diagnosed with UNE according to the criteria given above. The presence of denervation potentials, motor unit action potentials, and recruitment pathologies were recorded. Abnormal needle EMG findings were considered axonal damage. Needle EMG was conducted in muscles other than the ADM, FDI, and FCU for differential diagnosis when necessary.

\section{Statistical analysis}

Statistical analysis was performed using the statistical package SPSS software (Version 17.0, SPSS Inc., Chicago, IL, USA). The Kolmogorov Smirnov and Shapiro-Wilk tests and histograms were used to confirm normality of each continuous variable. Categorical variables between groups were analyzed using the Chi square test or Fisher's Exact Test.

The Student $\mathrm{t}$ test for normally distributed data was used for comparisons between groups. SPSS statistical software17.0 (SPSS Inc.) was used to construct receiver operating characteristic (ROC) curves and calculate the areas under curve (AUC), sensitivity, and specificity. P values of less than 0.05 were considered statistically significant.

\section{RESULTS}

Table 1 summarizes diagnoses resulting from ENMG findings. 
UNE was identified in only 11 of the 166 extremities. ENMG data of 114 patient extremities were completely normal. The remaining 41 extremities, which had results consistent with non-UNE ENMG pathologies, were not included in the results. Agreement between the prediagnosis and the electrophysiological diagnosis was $6.7 \%$ and the kappa coefficient was $0 \%$. Gender, age, height, weight, and body mass index of the two groups were similar ( $p>0.05)$. F wave latencies, sensory and motor NCV, all FIVD measurements, and CMAP amplitudes from the FDI muscle at the elbow segment were statistically different between the two groups $(\mathrm{p}<0.05)$. Distal latencies, CMAP amplitudes from the ADM muscle, and SNAP amplitudes from the elbow segments were similar $(p>0.05)$. Data of the two groups are summarized in Table 2.

In the UNE group, reduction of amplitude lower than $20 \%$ at the elbow segment from stimulus at the ADM and FDI was found to be in 100\% kappa agreement. For this evaluation, the sensitivity and specificity were $100 \%$.

UNE probability was determined to be $90.6 \%$ when the NCV value from FDI muscle was lower than $54.9 \mathrm{~m} / \mathrm{s}$ with a sensitivity of $99.1 \%$ and specificity of $81.8 \%$. For the ADM, the rate of accuracy was $93.8 \%$ with a cut-off value of $53.65 \mathrm{~m} / \mathrm{s}$. Sensitivity was $98.2 \%$ and specificity $90.9 \%$.

When sensorial FIVD was greater than $10.9 \mathrm{~m} / \mathrm{s}$, the probability of UNE was $88.9 \%$ at a sensitivity of $81.8 \%$ and specificity of $86.8 \%$. When the FIVD from stimulus at the ADM muscle was greater than $6.8 \mathrm{~m} / \mathrm{s}$, the probability of UNE was $80.1 \%$ with a sensitivity of $81.8 \%$ and specificity of78.1\%. The cut-off value of FIVD for the FDI muscle was $8.4 \mathrm{~m} / \mathrm{s}$ with a UNE probability of $86.9 \%$. The sensitivity and specificity for this evaluation was $72.7 \%$ and $90.2 \%$, respectively.

the other ENMG parameters sensitivity and specificity could not be calculated due to the lack of kappa agreement. 
Data of patients diagnosed with isolated UNE were statistically evaluated. Pathological ENMG findings of the UNE group can be found in Table 3.

\section{DISCUSSION}

Our ENMG results were within normal limits and the agreement between clinical prediagnosis and ENMG results was $6.7 \%$. Studies conducted in Turkey have reported a clinical pre-diagnosis-ENMG impressions agreement rate between $42.3 \%$ and $55 \%$ (7).

The extremely low agreement between clinical pre-diagnosis and ENMG results may suggest that inadequate clinical pre-diagnosis routinely leads to unnecessary ENMG requests. However, such a generalisation may not be correct. A study evaluating 130 cadavers reported that anatomical variations may create difficulty in determining causes of UNE (8). Within a peripheral nerve, nerve fibres innerving certain muscles are separated into different fascicles by the connective tissue. In the ulnar nerve, the nerve fibre bundle innerving the ADM muscle may be different than that innerving the FDI muscle and these nerve fibre bundles may be localised at different regions of the ulnar nerve. Thus, external pressure on the peripheral nerve may result in clinical and electrophysiological heterogeneity depending on the anatomical localisation at the site of pressure and the damage of the fascicles of the nerve $(1,2,9)$.

Stewart et al. (9) demonstrated that ulnar NCV in the same individual can vary by as much as $40 \mathrm{~m} / \mathrm{s}$. Such in accuracies are likely the result of several factors: (a) depolarization of the nerve some variable distance from the electrode, (b) shifts in electrode position due to skin laxity, and (c) the disproportionate effect of small errors in measurement due to the short 
distance between the proximal and distal stimulation sites. Our, results may have been affected by one or more of these factors.

Azma et al. (10) compared normal ulnar motor NCV values using the FDI and ADM on 100 nerves of healthy volunteers. Mean NCVs at the elbow were $62.65 \pm 7.62 \mathrm{~m} / \mathrm{s}$ and $60.49 \pm 7.42 \mathrm{~m} / \mathrm{s}$ for the ADM and FDI, respectively. Similarly, in the current study, NCV was $67.58 \pm 9.21 \mathrm{~m} / \mathrm{s}$ for the ADM and $66.39 \pm 9.05 \mathrm{~m} / \mathrm{s}$ for the FDI in non- UNE group.

Padua et al. reported that false-negatives were common with ENMG in cases of mild UNE (11). In the present study, CMAP amplitudes of the ADM and SNAP amplitude were similar between the two groups. Accordingly, false negatives may have occurred with mild UNE present in patients in the non-UNE group with reduced amplitude and without an obvious conduction block which could not be identified.

While the pathophysiology of many cases of UNE is of axonal loss, NCV demonstrates only a non-localizable ulnar neuropathy (1). In the present study, nonlocalizable ulnar neuropathy was detected in 13 cases. However, ENMG assessments of these 13 patients were not sufficient to verify UNE co-existing with axonal loss.

Studies in the literature have compared ENMG results from the ADM and FDI muscles of UNE patients. Kothari et al. (12) found that motor NCS of the FDI and ADM were abnormal in $81 \%$ and $71 \%$ of patients, respectively. Caliandro et al. (2) showed that FDI-innervated ulnar fibres have a higher susceptibility to damage than those of the ADM and reported sensitivity and specificity rates of $58 \%$ and $100 \%$, respectively, between the NCVs of both the FDI and ADM. Other studies have made similar findings $(9,13)$. Another study has suggested that recording from the FDI was not routinely indicated and that ADM recordings were sufficient for the diagnosis of UNE (5). Conversely, other studies argued that the ADM and FDI muscles were equally sensitive $(4,14)$. In the present study, a high rate of 
sensitivity and specificity was found in the FIDV, CMAP amplitude and motor NCV at the elbow segment. These sensitivity and specificity values were close for both muscles.

Kothari et al. (12) observed that the most common localizing finding of ENMG was conduction velocity slowing at the elbow segment. Findings in the current study (rate of slowing of NCV: $81.8 \%$ ) most resemble those of Kothari et al.

The FCU muscle may also be affected in UNE in agreement with its clinical and electrophysiological severity (1). In the present study, the determination rate of FCU impact by needle EMG was $27.27 \%$.

FIVD may be used in the diagnosis of UNE, with differences greater than $10 \mathrm{~m} / \mathrm{s}$ signalling UNE (3). Shakir et al.(6) compared motor NCV and FIVD of the ADM and FDI muscles of 85 subjects with UNE and 77 subjects with CTS without clinical evidence of UNE. At $95 \%$ specificity, motor NCV sensitivities were $80 \%$ at the ADM and $77 \%$ at the FDI, while FIVD sensitivities were $51 \%$ and $38 \%$, respectively. In the current study, FIVD was evaluated in both sensorial and motor measurements and sensorial and motor FIVDs had high sensitivity and specificity rates. However, while Shakir et al. divided the UNE group into three subgroups based on CMAP amplitude, we were unable to divide our groups based on CMAP amplitude and this difference in methodology should be considered when comparing these two studies.

The low number of UNE patients in accordance with the ENMG criteria can be considered a limitation of the present study and may affect the sensitivity and specificity reliability. In addition, the high number of patients with normal ENMG findings and the comparison between these two groups may have affected the present results. Studies with a higher number of patients with UNE diagnosis may contribute further to the literature.

In conclusion, the proportion of patients diagnosed with UNE that are in accordance with the existing electroneuromyographic criteria appears to be very low. According to our 
data, reduced CMAP amplitude has $100 \%$ sensitivity and specificity. In addition, elbow segment NCV from the ADM and FDI muscles have high sensitivity and specificity. However, sensitivity and specificity values were similar for the ADM and FDI muscles. Sensory and ADM/FDI FIVD may be useful for the diagnosis of UNE. 


\section{REFERENCES}

1. Preston DC, Shapiro B. Electromyography and neuromuscular disorders, clinicalelectrophysiologic correlations. Philadelphia: Elsevier; 2005: 291-312.

2. Caliandro P, Foschini M, Pazzaglia C, La Torre G, Aprile I, Granata G,et al. INRATIO: a new test to increase diagnostic sensitivity in ulnar nerve entrapment at elbow. Clin Neurophysiol 2008;119:1600-6.

3. American Association of Electrodiagnostic Medicine. Practice parameter for electrodiagnostic studies in ulnar neuropathy at the elbow: summary statement. American Association of Electrodiagnostic Medicine, American Academy of Neurology, American Academy of Physical Medicine and Rehabilitation. Muscle Nerve 1999;22:408-11.

4. Beekman R, Van Der Plas JP, Uitdehaag BM, Schellens RL, Visser LH. Clinical, electrodiagnostic, and sonographic studies in ulnar neuropathy at the elbow. Muscle Nerve 2004a;30:202-8.

5. Todnem K, Michler RP, Wader TE, Engstrøm M, Sand T. The impact of extended electrodiagnostic studies in ulnar neuropathy at the elbow. BMCNeurol 2009;9:52.

6. Shakir A,MicklesenPJ,Robinson LR. Which motor nerve conduction study is best in ulnar neuropathy at the elbow? Muscle Nerve 2004;29:585-90.

7. Yağcı I, Ofluoglu D, Gündüz H, KaradağSaygı E, Güven Z, Berker N,et al. The Consistency of Clinical Referral Diagnosis and Electrophysiological Diagnosis in Pediatric Cases Turk J Phys Med Rehab 2008;54:92-5.

8. Campbell WW, Pridgeon RM, Riaz G, Astruc J, Sahni KS. Variations in anatomy of the ulnar nerve at the cubital tunnel: pitfalls in the diagnosis of ulnar neuropathy at the elbow. Muscle Nerve 1991;14:733-8. 
9. Stewart JD. The variable clinical manifestations of ulnar neuropathies at the elbow. J Neurol Neurosurg Psychiatry 1987;50:252-8.

10. Azma K, Bahmanteimoury K, Tavana B, Moghaddam FR, Moghaddam NM, Mahmoudi H. Two measurement methods of motor ulnar nerve conduction velocity at the elbow: a comparative study. NeurolIndia2007;55:145-7.

11. Padua L, Aprile I, Mazza O, Padua R, Pietracci E, Caliandro P, et al. Neurophysiological classification of ulnar entrapment across the elbow. Neurol Sci 2001;22:11-6.

12. Kothari MJ, HeistandM, Rutkove SB. Three ulnar nerve conduction studies in patients with ulnar neuropathy at the elbow. Arch Phys Med Rehabil 1998;79:87-9.

13. Dumitru D, Zwarts MJ. Focal Peripheral Neuropathies.In: Dumitru D, Amato AA, Zwarts MJ, eds. Electrodiagnostic medicine. Hanley and Belfus Inc: Philadelphia; 2002:1076-81.

14. Tackmann W, Vogel P, Kaeser HE, Ettlin T. Sensitivity and localizing significance of motor and sensory electroneurographic parameters in the diagnosis of ulnar nerve lesions at the elbow. J Neurol 1984;231:204-11. 
Table 1: ElectoneuromyographicFindings

n

Normal
UNE
Non-localizable ulnar neuropathy
CTS + UNE
CTS
UNE+CTS+PNP
CTS + PNP
Non-localizable ulnar neuropathy + CTS
CTS+UNW
Mononeuritis multiplex
UNW
Motor neuron disease

Total

Abbrevations:UNE :Ulnar Neuropathy at the Elbow; UNW: Ulnar Neuropathy at theWrist; CTS: Carpal Tunnel Syndrome; PNP: Polyneuropathy
69.09

6.67

7.8

4.8

3.6

2.4

1.8

1.2

1.2

0.6

0.6

0.6

\section{6}


Table 2: Clinical and electroneuromyographiccharacteristics of groups (Mean \pm Standard deviation)

\begin{tabular}{|c|c|c|c|}
\hline & Group 1(UNE) & $\begin{array}{l}\text { Group } 2 \\
\text { (Non UNE) }\end{array}$ & $\mathbf{p}$ \\
\hline \multicolumn{4}{|l|}{ CLINICAL CHARACTERISTICS } \\
\hline Age (years) & $41.2 \pm 13.78$ & $42.52 \pm 12.41$ & 0.753 \\
\hline Body length(cm) & $168.4 \pm 7.56$ & $162.32 \pm 27.67$ & 0.493 \\
\hline Weight $(\mathrm{kg})$ & $73.1 \pm 18.14$ & $74.15 \pm 13.12$ & 0.82 \\
\hline Body Mass Index $\left(\mathrm{kg} / \mathrm{m}^{2}\right)$ & $25.54 \pm 5.04$ & $26.74 \pm 4.31$ & 0.42 \\
\hline \multicolumn{4}{|l|}{ ELECTRONEUROMYOGRAPHIC RESULTS } \\
\hline Minimum $F$ wave latency $(\mathrm{ms})$ & $26.31 \pm 2.29$ & $24.89 \pm 1.62$ & 0.009 \\
\hline Mean F wave latency(ms) & $27.54 \pm 2.16$ & $25.95 \pm 1.69$ & 0.005 \\
\hline \multicolumn{4}{|l|}{ Ulnar nerve sensory measurements } \\
\hline Distal latency (ms) & $2.85 \pm 2.22$ & $2.69 \pm 0.31$ & 0.107 \\
\hline SNAP Amplitude at the elbow (mV) & $11.91 \pm 8.25$ & $16.36 \pm 8.71$ & 0.107 \\
\hline $\mathrm{NCV}$ at the elbow segment $(\mathrm{m} / \mathrm{s})$ & $46.54 \pm 9.89$ & $70.06 \pm 10.84$ & $\mathbf{0 . 0}$ \\
\hline $\mathrm{NCV}$ at the forearm segment $(\mathrm{m} / \mathrm{s})$ & $64.81 \pm 6.4$ & $65.7 \pm 6.42$ & 0.661 \\
\hline FIVD & $18.27 \pm 12.35$ & $-4.36 \pm 14.57$ & $\mathbf{0 . 0}$ \\
\hline \multicolumn{4}{|c|}{ Ulnar nerve motor measurements of abductor digitiminimi muscle } \\
\hline Distal latency (ms) & $2.61 \pm 0.34$ & $2.58 \pm 0.24$ & 0.752 \\
\hline CMAP Amplitude at the elbow (mV) & $7.8 \pm 2.17$ & $8.96 \pm 2.42$ & 0.131 \\
\hline $\mathrm{NCV}$ at the elbow segment $(\mathrm{m} / \mathrm{s})$ & $49.6 \pm 10.07$ & $67.58 \pm 9.21$ & $\mathbf{0 . 0}$ \\
\hline $\mathrm{NCV}$ at the forearm segment $(\mathrm{m} / \mathrm{s})$ & $63.31 \pm 7.62$ & $64.96 \pm 5.62$ & 0.371 \\
\hline FIVD & $13.7 \pm 15.26$ & $-2.61 \pm 11.26$ & $\mathbf{0 . 0}$ \\
\hline \multicolumn{4}{|c|}{ Ulnar nerve motor measurements of first dorsal interosseous muscle } \\
\hline Distal latency (ms) & $3.0 \pm 0.32$ & $3.04 \pm 0.27$ & 0.598 \\
\hline CMAP Amplitude at the elbow $(\mathrm{mV})$ & $6.02 \pm 2.45$ & $7.73 \pm 2.63$ & 0.042 \\
\hline $\mathrm{NCV}$ at the elbow segment $(\mathrm{m} / \mathrm{s})$ & 47.989 .44 & $66.39 \pm 9.05$ & 0.0 \\
\hline $\mathrm{NCV}$ at the forearm segment $(\mathrm{m} / \mathrm{s})$ & $59.9 \pm 5.13$ & $61.52 \pm 5.77$ & 0.370 \\
\hline FIVD & $11.92 \pm 11.91$ & $-4.86 \pm 10.94$ & $\mathbf{0 . 0}$ \\
\hline
\end{tabular}

Abbrevations: NCV: Nerve Conduction Velocity; CMAP: Compound Muscle Action Potential; SNAP: Sensory Nerve Action Potential; FIVD: Forearm-elbow Inter-segmental Velocity Difference 
Table 3: Classification of pathological ENMG findings in the UNE group

\begin{tabular}{ll}
\hline $\mathbf{n}(11)$ & $\%$ \\
\hline
\end{tabular}

Axonal Damage based on Needle EMG

$\begin{array}{lll}\text { FDI } & 3 & 27.27 \\ \text { ADM } & 3 & 27.27 \\ \text { FCU } & 3 & 27.27\end{array}$

Conduction Block

$\begin{array}{lll}\text { Ulnar nerve / Sensory } & 4 & 36.36 \\ \text { Ulnar nerve / FDI motor } & 1 & 9.09 \\ \text { Ulnar nerve / ADM motor } & 1 & 9.09\end{array}$

Focal demyelination

Ulnar nerve / Sensory

$8 \quad 72.72$

Ulnar nerve / FDI motor

$9 \quad 81.81$

Ulnar nerve / ADM motor

$7 \quad 62.63$

ENMG: Electroneuromyography; UNE: Ulnar Neuropathy at the Elbow ADM:Muscle of Abductor Digiti Minimi; FDI: Muscle of First Dorsal Interosseous; FCU: Muscle of Flexor Carpi Ulnaris 